in vivo $34: 2009-2014(2020)$

doi:10.21873/invivo.11999

\title{
High-dose Radiotherapy for Oligo-progressive NSCLC Receiving EGFR Tyrosine Kinase Inhibitors: Real World Data
}

\author{
MARIACARMELA SANTARPIA ${ }^{1}$, GIUSEPPE ALTAVILLA ${ }^{1}$, NICOLO BORSELLINO ${ }^{2}$, \\ ANDREA GIRLANDO ${ }^{3}$, GIANFRANCO MANCUSO ${ }^{4}$, STEFANO PERGOLIZZI $^{5}$, DARIO PIAZZA ${ }^{6}$, \\ ANTONIO PONTORIERO ${ }^{5}$, MARIA ROSARIA VALERIO ${ }^{7}$ and VITTORIO GEBBIA ${ }^{4,8}$ \\ ${ }^{1}$ Medical Oncology Unit, Depart. of Human Pathology "G. Barresi”, University of Messina, Messina, Italy; \\ ${ }^{2}$ Medical Oncology Unit, Ospedale Buccheri La Ferla, Palermo, Italy; \\ ${ }^{3}$ Radiotherapy Unit, Istituto Clinico Humanitas, Catania, Italy; \\ ${ }^{4}$ Medical Oncology and Supportive Care Unit, La Maddalena Cancer Center, Palermo, Italy; \\ ${ }^{5}$ Unit of Radiation Oncology, Department of Biomedical, Dental Science and Morphological \\ and Functional Images, University of Messina, Messina, Italy; \\ ${ }^{6}$ GSTU Foundation for Cancer Research, Palermo, Italy; \\ ${ }^{7}$ Medical Oncology Unit, Department of Oncology, University of Palermo, Palermo, Italy; \\ ${ }^{8}$ PROMISE Department, University of Palermo, Palermo, Italy
}

\begin{abstract}
Background/Aim. Local ablative treatments for oligo-progressive, EGFR mutated non-small cell lung cancer (mut-NCSLC) may improve long-term disease control and survival. We analyzed the efficacy of hypo-fractionated, highdose radiation therapy (HDRT), in association with prolonged EGFR tyrosine kinase inhibitors (TKI) in oligo-progressive, EGFR mutant-NSCLC. Patients and Methods. Progressionfree survival-1 (PFS-1, date from initiation of TKI therapy until oligo-progression or death), and progression-free survival-2 (PFS-2, date of focal progression until further progression or death) were evaluated. Results. Thirty-six patients were analyzed. The median PFS 1 was 12.5 months. HDHRT consisted of intensity-modulated RT and stereotactic $R T$ in 23 (64\%) and 13 (36\%) patients respectively. The median PFS 2 was 6.3 months. Overall survival was 38.7 months. Conclusion. Hypo-fractionated HDRT plus TKI therapy, is associated with a significant prolongation of disease control (overall PFS: 18.8 months), with manageable side effects. These real-world data support the use of local ablative approaches in oligo-progressive EGFR mut-NSCLC.
\end{abstract}

This article is freely accessible online.

Correspondence to: Prof. Vittorio Gebbia, Medical Oncology Unit, La Maddalena Cancer Center, and PROMISE Department University of Palermo, Via S. Lorenzo, 312/D, 90146 Palermo, Italy. Tel: +39 330696205, e-mail: vittorio.gebbia@gmail.com

Key Words: Non-small cell lung cancer, EGFR, oligo-progression, high-dose radiotherapy.
Non-small cell lung cancer (NSCLC) is the most frequent form of lung cancer and is commonly diagnosed at locally advanced or metastatic stages (1). Systemic therapies for advanced NSCLC (aNSCLC) have markedly evolved during the last years due to the increasing number of molecularly targeted agents approved for the treatment of patients whose tumors harbor specific oncogenic alterations, such as mutations of EGFR, and ALK or ROS1 rearrangements, and to the advent of the immune checkpoint inhibitors, including monoclonal antibodies directed against the PD1-PD-L1 pathway $(2,3)$.

Approximately $25 \%$ of aNSCLC patients may present at diagnosis with oligometastatic disease, which is characterized by the presence of metastatic lesions limited in number and location. Although oligo-metastases has not been defined (4-7), the oligometastatic state is described as "an intermediate clinical state between locally confined and widely disseminated metastatic cancer" (8). In the real world, this term encompasses a broad spectrum of clinical entities, with varying numbers and sites of metastatic lesions, for which the optimal therapeutic strategies have not yet been determined. Oligometastatic tumors present a more indolent biology and have not yet gained the ability to spread to multiple organs, suggesting that, despite being metastatic, they can be associated with favorable prognosis. This observation is also underlined by the last edition of the TNM staging system by the International Association for the Study of Lung Cancer (IASLC), in which the M1b includes a single extrathoracic metastatic lesion versus widespread extra thoracic M1c disease (9). Oligo-progression is very common in oncogene-addicted aNSCLC. Indeed, despite the 
remarkable clinical benefit with EGFR- or ALK-tyrosine kinase inhibitors (TKI), acquired resistance invariably develops and represents a significant challenge to the durable efficacy of these agents.

Recent clinical data suggest that curative therapeutic approaches, including local ablative therapies, such as surgery and/or radiotherapy, should be considered for patients with oligometastatic tumors at diagnosis, to improve disease control and thereby long-term survival outcomes (7). Progression in few metastatic sites under TKI therapy may be postulated to be a result of clonal evolution through the accumulation of molecular alterations in cancer cells, which can render some sub-clones resistant. In contrast, others remain still controlled on continued systemic targeted therapy $(10,11)$. The most common mechanism of resistance to first- and second-generation EGFR TKIs is the secondary, gatekeeper T790M mutation in exon 20 of EGFR that has been reported in approximately $50-60 \%$ of EGFR TKIresistant tumors and can be currently targeted by the thirdgeneration TKI osimertinib $(11,12)$. The mechanisms of resistance to targeted drugs may be different, and multiple sub-clones with distinct molecular profiles and drugsensitivity can coexist within the primary tumor or at different metastatic sites.

Continuation of TKI treatment beyond progression in patients with oligo-progressive aNSCLC can be an effective strategy to target tumor cells that remain still addicted to the EGFR pathway and prevent the disease flare observed after discontinuation of the targeted agent $(13,14)$. As recently shown, this strategy can delay the initiation of more toxic and less-tolerated second-line therapy, such as chemotherapy, in patients with indolent or asymptomatic progression (15). In addition, local ablative therapies to metastatic sites can further improve long-term disease control. Stereotactic body radiotherapy (SBRT) or SBRT-like treatments have been used in patients with oligometastatic or oligo-progressive tumors, including aNSCLC, to treat metastasis at different anatomical sites, with encouraging results in terms of rates of local control rate and overall survival and limited toxicity $(7,16-22)$.

In this study, we retrospectively evaluated the feasibility and efficacy of the addition of high-dose hypo-fractionated HDRT to post-progression TKI therapy in EGFR-mutant NSCLC patients with oligo-progressive disease.

\section{Patients and Methods}

This retrospective analysis included all patients with histologically confirmed aNSCLC harboring EGFR mutations who developed oligo-progression during first-line TKI therapy and subsequently treated with hypo-fractionated HDRT to the sites of progression. After approval of the study by the Ethics Committee, patient data were obtained anonymously from the electronic records in respect of the Declaration of Helsinki. Written informed consent was obtained from all patients to receive targeted therapy and
Table I. Clinical and demographic characteristics of patients.

\begin{tabular}{lc}
\hline Number of enrolled patients & $36(100 \%)$ \\
Median age and range (years) & $66(52-77)$ \\
Gender (males/female) & $24(66 \%) / 12(34 \%)$ \\
Never smokers & $23(64 \%)$ \\
Adenocarcinoma & $36(100 \%)$ \\
EGFR mutation & $36(100 \%)$ \\
Exon 19 del & $22(61 \%)$ \\
Exon 21 (L858R) & $13(36 \%)$ \\
Others (G719X) & $1(3 \%)$ \\
Previous treatments & \\
$\quad$ Surgery & $2(5 \%)$ \\
Radiotherapy & $1(2.5 \%)$ \\
\hline
\end{tabular}

radiotherapy. The need for patients' informed consent was waived due to the retrospective nature of the study.

Criteria for patients' data selection included the presence of diagnostic tumor specimens positive for EGFR mutation status by real-time PCR with a validated test kit (TheraScreen EGFR RGQ PCR Kit, Qiagen, Manchester, UK), and complete follow-up data and treatment-related toxicity records. The collected data included age at diagnosis, sex, tumor histology, prior therapy, smoking status (categorized as current: smoked within $<1$ year before the initiation of therapy, former: quit $\geq 1$ year before the initiation of therapy, or never: $<100$ lifetime cigarettes), pre-treatment complete radiologic staging (by total body computed tomography-CT scan and/or positron emission tomography-PET/CT scan), sites of disease and concomitant non-neoplastic disorders.

Treatment consisted of oral first-line gefitinib at the dose of $250 \mathrm{mg}$ per day. Clinical examination and complete blood testing were performed before each cycle (every 30 days). According to the Italian regulatory drug agency indications and routine institutional practice, the first radiologic assessment was performed 2-3 months after initiating treatment and subsequently. Tumor response was assessed according to the RECIST criteria version 1.1 on chest and abdominal $\mathrm{CT}$ or PET scans. Patients developing oligo-progressive disease were evaluated for possible local treatments by a multidisciplinary team, including medical oncologists, radiotherapists, thoracic surgeons, radiologists, and pneumologists. Hypo-fractionated HDRT consisted of intensity-modulated RT (IMRT) and stereotactic radiotherapy (SRS and SBRT). The number and sites of metastatic disease, as well as dosage and timing of HDRT, were carefully recorded. Patients continued gefitinib at the same dose during radiotherapy, and they were carefully monitored for any adverse events. Progression-free survival (PFS) was first calculated from the date of the initiation of gefitinib until documentation of oligo-progression of disease or death for any cause (PFS-1), and from the date of limited progression until further progression of disease or death (PFS-2). RECIST criteria defined progression of the disease. PFS and OS analysis were determined according to the Kaplan-Meier product limit analysis, and curves performed using Prism IV software (GraphPad, San Diego, CA, USA).

\section{Results}

Study design. We reviewed data from advanced EGFRmutant aNSCLC patients treated with gefitinib between 
Table II. Progression-free survival 1 and site of first oligo-progression.

\begin{tabular}{lc}
\hline PFS 1 median and range (months)* & $12.5(4.0-23.2)$ \\
Site of first oligo-progression (n. of patients) & \\
Lung** & $11(30.5 \%)$ \\
Node & $9(25 \%)$ \\
CNS $^{\wedge}$ & $9(25 \%)$ \\
Bone & $5(14 \%)$ \\
Adrenal & $2(5.5 \%)$ \\
\hline
\end{tabular}

*PFS-1: First progression free-survival according to RECIST criteria or clinical assessment; ${ }^{* *}$ other than primary tumor; ${ }^{\wedge}$ number of lesions $\leq 3$.

January 2012 and December 2018 and identified 36 patients who satisfied the inclusion criteria for this study. Patients progressing on gefitinib but with poor tolerance or had more than 3 metastatic sites of CNS progression or leptomeningeal disease or had poor performance status $(\mathrm{ECOG} \geq 2)$ were excluded from local treatment.

Patient population. The patients' characteristics are listed in Table I. All patients had adenocarcinoma. EGFR mutations were detected in all tumor specimens and included deletion of exon 19 in $22(61 \%)$ patients, L858R mutation in exon 21 in 13 patients (36\%), and an uncommon mutation in only 1 case (G719X mutation in exon 18). Overall, 24 patients $(66 \%)$ were male, and $13(34 \%)$ females with a median age of 66 years (range $=52-77$ years). Most patients (23/36: 64\%) were never smokers. All patients received gefitinib at a standard dose of $250 \mathrm{mg} /$ day as first-line therapy.

Outcomes. Objective responses at first assessment were observed in 21 patients $(58.3 \%)$, and all were partial responses. Stabilization of disease was observed in 14 cases $(38.9 \%)$ and progressive disease in 1 case. The overall median PFS-1, measured from the initiation of front-line TKI therapy until oligo-progression, was 12.5 months (range $=4.0$ 23.2 months) (Table II; Figure 1). As shown in Table II, the most common site of oligo-progression was lung (30.5\%), followed by nodes $(25 \%)$, CNS $(25 \%)$, bone $(14 \%)$ and adrenals $(5.5 \%)$. All 36 patients received a local treatment consisting of hypo-fractionated HDRT to the site of focal progression which consisted of intensity-modulated RT in 23 patients $(64 \%)$ and SBRT in 13 cases $(36 \%)$, with a median delivered dose of $30 \mathrm{~Gy}$ (range $=12-60 \mathrm{~Gy}$ ), and a median number of fractions of 10 (range $=2-30$ fractions). The median time between the diagnosis of progression and the initiation of HDRT was 22 days. The majority of patients were treated to a single metastatic site $(22 / 36 ; 61 \%)$, while 10 patients $(28 \%)$ and 4 patients $(11 \%)$ received local treatment to 2 and 3 sites, respectively.

As shown in Figure 1 and in Table III, the median PFS 2 was 6.3 months (range=2-12.5 months). Metastatic deposits

\section{PFS-1 and PFS1 + PFS-2}

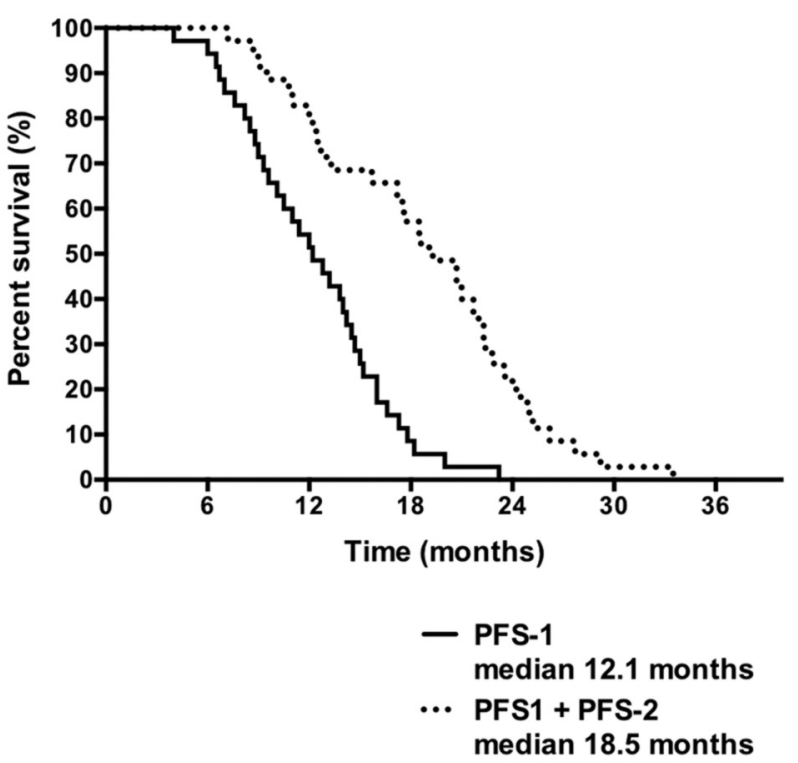

Figure 1. Progression-free survival (PFS)-1 and -2 .

Table III. Number of irradiated sites and progression-free survival (PFS)-1 ad -2.

\begin{tabular}{lc} 
Number of enrolled patients & $36(100 \%)$ \\
Number of metastatic sites treated & \\
1 Site & $22(61 \%)$ \\
2 Sites & $10(28 \%)$ \\
3 Sites & $4(11 \%)$ \\
PFS 2 median and range (months)* & $6.3(2.0-12.5)$ \\
PFS 1+PFS 2 median and range (months) & $18.5(8.0-33.5)$ \\
Site of second progression (n. of patients) & $16(44 \%)$ \\
Lung** & $13(36 \%)$ \\
Node & $12(33 \%)$ \\
CNS & $8(22 \%)$ \\
Bone & $3(8 \%)$ \\
Adrenal & \\
\hline
\end{tabular}

*PFS-1: First progression free-survival according to RECIST criteria or clinical assessment; **other than primary tumor; ${ }^{\wedge}$ number of lesions $\leq 3$.

treated with hypo-fractionated HDRT represented the site of second-progression only in 6 patients $(16.7 \%)$, indicating a high rate of durable control in the irradiated sites.

No correlation between EGFR mutation status and outcome data was observed. Type of mutation showed no correlation either with the response to hypo-fractionated HDRT or PSF-2 $(r=0.103, p=0.725)$. At the time of secondary progression, nine patients $(25 \%)$ showing T790M mutation received osimertinib. Seven patients (19.4\%) were 
kept on gefitinib until symptomatic clinical progression started to appear and therefore treated with pemetrexed and platinum salts. Globally, OS was 38.7 months (range=9.046.3 months).

Toxicity. Treatment with gefitinib was administered continuously during radiation therapy. Overall, during the whole treatment with gefitinib, the most frequent treatmentrelated adverse events were mainly of grade 1 and 2; grade $3 \mathrm{rash} / \mathrm{acne}$ was observed in 1 case $(2.7 \%)$, and transitory grade 2 elevated transaminases was recorded in 2 cases (5.5\%). No $\geq 4$-grade toxicities were reported, and no patient had to discontinue TKI therapy because of adverse effects during radiotherapy. No case of severe pulmonary toxicity or radiation-related necrosis was reported.

\section{Discussion}

EGFR-mutant aNSCLC represents a unique disease for which several molecularly targeted drugs are currently available. First and second-generation EGFR TKIs have been widely demonstrated to prolong survival and improve quality of life compared to standard chemotherapy in the first-line setting. However, chronic exposure to TKI may ultimately induce the development of acquired resistance, which finally limits the long-term efficacy of these drugs. In patients with advanced mutated NSCLC, oligo-progression during TKI therapy is common $(7,8)$.

Several therapeutic strategies have been evaluated, including the continuation of a targeted agent beyond objective progression and the addition of local ablative treatment, such as surgery and/or radiation therapy, to limited metastatic sites $(7,19-25)$. The rationale of this approach is that a definitive local therapy can effectively target TKIresistant clones in progressive sites of disease, while the continuation of the TKI can be useful to maintain the EGFR pathway blockade in the remainder sensitive clones that have not yet acquired resistance.

Most of the reported studies on the benefits of RT techniques in oligo-progressive NSCLC patients are retrospective and included small and heterogeneous cohorts of patients (19-26). In a phase II study, unselected NSCLC patients with the oligo-progressing disease ( $\leq 6$ extra-cranial metastatic sites) during first-line chemotherapy were treated with concomitant erlotinib and SBRT directed to all sites of progressive disease (21). Median PFS was 14.7 months and a median OS 20.4 months. This combined approach was well-tolerated, resulting in only two SBRT-related grade 3 toxicities among 24 patients enrolled (19). In another retrospective analysis of 15 cases, definitive SBRT and continuation of TKIs provided a long-lasting disease control, with the median PFS 2 and OS of 10.9 months and 39 months respectively (20). Weickhardt et al. have reported the use of local ablative therapies in EGFR-mutant or ALKrearranged NSCLC patients treated beyond progression with erlotinib or crizotinib, respectively (21). This strategy was associated with more than 6 months of additional disease control after progression, with a median PFS-2 of 6.2 months and no severe RT-related toxicity (21). Yu et al. retrospectively analyzed data of 18 patients with mutNSCLC patients who received local elective therapy, including surgical resection, radio-frequency ablation, or radiation (22). An encouraging median PFS-2 of 10 months and median OS 41 months were observed. Notably, in those patients who were positive for T790M, osimertinib can be still administered at further progression after local ablative therapies, thus conferring an additional PFS advantage. In our series, due to regulatory affairs, only 9 patients received the third-generation TKI.

In our study, we analyzed the efficacy and safety of a therapeutic strategy based on continued EGFR inhibition by gefitinib in association with hypo-fractionated HDRT to limited sites of disease, in patients with oligo-progressive advanced, EGFR-mutated NSCLC. In our hands, this approach is feasible, highly effective, and well-tolerated with PFS-1 of 12.5, PFS-2 6.3 months, and an overall PFS of 18.8 months. Our results are in agreement with those reported in the literature (21-27). The toxicity profile of our approach was good, with no grade $\geq 4$ toxicities, suggesting that the continuation of gefitinib did not increase radiation-related potential adverse events, including pneumonitis.

Despite the efficacy of local ablative therapies, the exact number of metastatic sites that can be treated to obtain a long-term clinical benefit has not yet been established. A meta-analysis including 757 mutation unselected, oligometastatic NSCLC patients with one metachronous metastasis treated with ablative treatments has reported a favorable 5-year OS rate of $29.4 \%$ (5). Another systematic review of retrospective observational cohort studies included 668 patients with an oligometastatic disease with up to 5 synchronous metastases, reported single-organ involvement, and the number of brain metastases as an independent prognostic factor for survival $(27,28)$. As suggested by other authors, one of the advantages of this approach is the possibility to extend TKI therapy beyond objective progression and delay the start of more toxic and less tolerated systemic treatment particularly in patients with a longer time to progression, a slow rate of progression and lack of new extra-thoracic metastases $(14,29)$. Another possible limitation of this study is linked to the difficulty in predicting the risk of widely metastatic dissemination. Therefore, despite modern radiation techniques that can technically treat an increasing number of synchronous metastatic sites, this approach may not necessarily translate into improved clinical outcomes. However, in a prospective phase II study including patients who did not progress to 
first-line therapy, including chemotherapy, EGFR or ALK inhibitors, and had $\leq 3$ metastatic sites, consolidation therapy with radiotherapy or surgery increased PFS compared to maintenance therapy (which could be observation only). Despite some limitations, such as the small number and molecular heterogeneity of patients enrolled, these results suggest a potential role for local therapies also in an earlier clinical setting of patients who have not yet developed progressive disease (30).

In conclusion, incorporation of hypo-fractionated HDRT into the management of oligo-metastatic and oligoprogressive patients with mut-NSCLC can be a reasonable therapeutic option being able to improve long-term disease control with low toxicity.

\section{Conflicts of Interest}

All Authors declare that they have no competing interests related to this study.

\section{Authors' Contributions}

Study concept and design: MS and VG. Acquisition, analysis, and interpretation of data: MS, MRV, NB, AG, GA, GM, SP, AP and VG. Drafting of the manuscript, statistical analyses: MS, DP and VG. Critical revision of the manuscript: all Authors. All Authors read and approved the final manuscript.

\section{References}

1 Ferlay J, Colombet M, Soerjomataram I, Mathers C, Parkin DM, Piñeros M, Znaor A and Bray F: Estimating the global cancer incidence and mortality in 2018: GLOBOCAN sources and methods. Int J Cancer 144(8): 1941-1953, 2019. PMID: 30350310. DOI: $10.1002 /$ ijc. 31937

2 Hirsch FR, Scagliotti GV, Mulshine JL, Kwon R, Curran WJ Jr, Wu YL and Paz-Ares L: Lung cancer: current therapies and new targeted treatments. Lancet 389(10066): 299-311, 2017. PMID: 27574741. DOI: 10.1016/S0140-6736(16)30958-8

3 Planchard D, Popat S, Kerr K, Novello S, Smit EF, Faivre-Finn C, Mok TS, Reck M, Van Schil PE, Hellmann MD and Peters S: Metastatic non-small cell lung cancer: ESMO Clinical Practice Guidelines for diagnosis, treatment and follow-up. Ann Oncol 29: iv192-iv237, 2018. PMID: 31987360. DOI: 10.1093/ annonc/mdy 474

4 Ashworth A, Rodrigues G, Boldt G and Palma D: Is there an oligometastatic state in non-small cell lung cancer? A systematic review of the literature. Lung Cancer 82: 197-203, 2013. PMID: 24051084. DOI: 10.1016/j.lungcan.2013.07.026

5 Ashworth AB, Senan S, Palma DA, Riquet M, Ahn YC, Ricardi U, Congedo MT, Gomez DR, Wright GM, Melloni G, Milano MT, Sole CV, De Pas TM, Carter DL, Warner AJ and Rodrigues GB: An individual patient data meta-analysis of outcomes and prognostic factors after treatment of oligometastatic non-smallcell lung cancer. Clin Lung Cancer 15: 346-355, 2014. PMID: 24894943. DOI: 10.1016/j.cllc.2014.04.003

6 Parikh RB, Cronin AM, Kozono DE, Oxnard GR, Mak RH, Jackman DM, Lo PC, Baldini EH, Johnson BE and Chen AB:
Definitive primary therapy in patients presenting with oligo metastatic non-small cell lung cancer. Int J Radiat Oncol Biol Phys 89: 880-887, 2014. PMID: 24867533. DOI: 10.1016/ j.ijrobp.2014.04.007

7 Stephens SJ, Moravan MJ and Salama JK: Managing patients with oligometastatic non-small-cell lung cancer. J Oncol Pract 14(1): 23-31, 2018. PMID: 29324212. DOI: 10. 1200/JOP. 2017.026500

8 Weichselbaum RR and Hellman S: Oligometastases revisited. Nat Rev Clin Oncol 8(6): 378-382, 2011. PMID: 21423255. DOI: $10.1038 /$ nrclinonc.2011.44

9 Eberhardt WEE, Mitchell A, Crowley J, Kondo H, Kim YT, Turrisi A 3rd, Goldstraw P and Rami-Porta R; International Association for Study of Lung Cancer Staging and Prognostic Factors Committee, Advisory Board Members, and Participating Institutions. The IASLC Lung Cancer Staging Project: Proposals for the Revision of the M Descriptors in the Forthcoming Eighth Edition of the TNM Classification of Lung Cancer. J Thorac Oncol 10(11): 1515-1522, 2015. PMID: 26536193. DOI: 10.1097/JTO.0000000000000673.

10 Amirouchene-Angelozzi N, Swanton C and Bardelli A: Tumor evolution as a therapeutic target. Cancer Discov 7: 805-817, 2017. PMID: 28729406. DOI: 10.1158/2159-8290.CD-17-0343.

11 Santarpia M, Gil N and Rosell R: Strategies to overcome resistance to tyrosine kinase inhibitors in non-small-cell lung cancer. Expert Rev Clin Pharmacol 8(4): 461-477, 2015. PMID: 26068305. DOI: $10.1586 / 17512433.2015 .1055252$

12 Santarpia M, Liguori A, Karachaliou N, Gonzalez-Cao M, Daffinà MG, D'Aveni A, Daffinà MG, Lazzari C, Altavilla G and Rosell R: Osimertinib in the treatment of non-small-cell lung cancer: design, development and place in therapy. Lung Cancer (Auckl) 8: 109-125, 2017. PMID: 29780635. DOI: $10.21037 /$ jtd .2018 .03 .81

13 Chaft JE, Oxnard GR, Sima CS, Kris MG, Miller VA and Riely GJ: Disease flare after tyrosine kinase inhibitor discontinuation in patients with EGFR-mutant lung cancer and acquired resistance to erlotinib or gefitinib: implications for clinical trial design. Clin Cancer Res 17: 6298-303, 2011. PMID: 21856766. DOI: 10.1158/1078-0432.CCR-11-1468

14 Camidge DR, Pao W and Sequist LV: Acquired resistance to TKIs in solid tumours: learning from lung cancer. Nat Rev Clin Oncol 11(8): 473-448, 2014. PMID: 24981256. DOI: 10.1038/ nrclinonc.2014.104

15 Park K, Yu CJ, Kim SW, Lin MC, Sriuranpong V, Tsai CM, Lee JS, Kang JH, Chan KC, Perez-Moreno P, Button P, Ahn MJ and Mok T: First-line erlotinib therapy until and beyond response evaluation criteria in solid tumors progression in asian patients With Epidermal Growth Factor Receptor Mutation-Positive NonSmall-Cell Lung Cancer: The ASPIRATION Study. JAMA Oncol 2(3): 305-312, 2016. PMID: 26720423. DOI: 10.1001/ jamaoncol. 2015.4921

16 Lewis SL, Porceddu S, Nakamura N, Palma DA, Lo SS, Hoskin P, Moghanaki D, Chmura SJ and Salama JK: Definitive stereotactic body radiotherapy (SBRT) for extracranial oligometastases: an international survey of $>1000$ radiation oncologists. Am J Clin Oncol 40: 418-422, 2015. PMID: 25647831. DOI: 10.1097/COC.0000000000000169

17 Bergsma DP, Salama JK, Singh DP, Chmura SJ and Milano MT: Radiotherapy for oligometastatic lung cancer. Front Oncol 7: 210, 2017. PMID: 28975081. DOI: 10.3389/fonc.2017.00210 
18 Juan $\mathrm{O}$ and Popat S: Ablative therapy for oligometastatic nonsmall cell lung cancer. Clin Lung Cancer 18(6): 595-606, 2017. PMID: 28377206. DOI: 10.1016/j.cllc.2017.03.002

19 Iyengar P, Kavanagh BD, Wardak Z, Smith I, Ahn C, Gerber DE, Dowell J, Hughes R, Abdulrahman R, Camidge DR, Gaspar LE, Doebele RC, Bunn PA, Choy H and Timmerman R: Phase II trial of stereotactic body radiation therapy combined with erlotinib for patients with limited but progressive metastatic non-smallcell lung cancer. J Clin Oncol 32: 3824-3830, 2014. PMID: 25349291. DOI: $10.1200 /$ JCO.2014.56.7412

20 Conforti F, Catania C, Toffalorio F, Duca M, Spitaleri G, Barberis M, Noberasco C, Delmonte A, Santarpia M, Lazzari C and De Pas TM: EGFR tyrosine kinase inhibitors beyond focal progression obtain a prolonged disease control in patients with advanced adenocarcinoma of the lung. Lung Cancer 81(3): 440444, 2013. PMID: 23810573. DOI: 10.1016/j.lungcan. 2013.05.019

21 Weickhardt AJ, Scheier B, Burke JM, Gan G, Lu X, Bunn PA Jr, Aisner DL, Gaspar LE, Kavanagh BD, Doebele RC and Camidge DR: Local ablative therapy of oligoprogressive disease prolongs disease control by tyrosine kinase inhibitors in oncogene-addicted non-small-cell lung cancer. J Thorac Oncol 7: 1807-1814, 2012. PMID: 23154552. DOI: 10.1097/JTO. 0b013e3182745948

22 Yu HA, Sima CS, Huang J, Solomon SB, Rimner A, Paik P, Pietanza MC, Azzoli CG, Rizvi NA, Krug LM, Miller VA, Kris MG and Riely GJ: Local therapy with continued EGFR tyrosine kinase inhibitor therapy as a treatment strategy in EGFR-mutant advanced lung cancers that have developed acquired resistance to EGFR tyrosine kinase inhibitors. J Thorac Oncol 8(3): 346-351, 2013. PMID: 23407558. DOI: 10.1097/JTO. 0b013e31827e1f83

23 Ning MS, Gomez DR, Heymach JV and Swisher SG: Stereotactic ablative body radiation for oligometastatic and oligoprogressive disease. Transl Lung Cancer Res 8(1): 97-106, 2019. PMID: 30788239. DOI: 10.21037/tlcr.2018.09.21

24 Kroeze SG, Fritz C, Hoyer M, Lo SS, Ricardi U, Sahgal A, Stahel R, Stupp R and Guckenberger M: Toxicity of concurrent stereotactic radiotherapy and targeted therapy or immunotherapy: A systematic review. Cancer Treat Rev 53: 2537, 2017. PMID: 28056412. DOI: 10.1016/j.ctrv.2016. 11.013

25 Pontoriero A, Conti A, Iatì G, Mondello S, Aiello D, Rifatto C, Risoleti E, Mazzei M, Tomasello F, Pergolizzi S and De Renzis C: Prognostic factors in patients treated with stereotactic imageguided robotic radiosurgery for brain metastases: a single-center retrospective analysis of 223 patients. Neurosurg Rev 39(3): 495-504, 2016. PMID: 27106896. DOI 10.1007/s10143-0160718-7
26 Tree AC, Khoo VS, Eeles RA, Ahmed M, Dearnaley DP, Hawkins MA, Huddart RA, Nutting CM, Ostler PJ and van As NJ: Stereotactic body radiotherapy for oligometastases. Lancet Oncol 14: e28-37, 2013. PMID: 23276369. DOI: 10.1016/ S1470-2045(12)70510-7

27 Li D, Zhu X, Wang H, Qiu M and Li N: Should aggressive thoracic therapy be performed in patients with synchronous oligometastatic non-small cell lung cancer? A meta-analysis. J Thorac Dis 9: 310-317, 2017. PMID: 28275479. DOI: 10.21037/ jtd.2017.02.21

28 Pessina F, Navarria P, Cozzi L, Tomatis S, Ascolese AM, Franzese C, Toschi L, Santoro A, De Rose F, Franceschini D, Bello L and Scorsetti M: Outcome appraisal of patients with limited brain metastases (BMs) from non-small cell lung cancer (NSCLC) treated with different local therapeutic strategies: a single institute evaluation. Br J Radiol 90: 20170022, 2017. PMID: 28256924. DOI: 10.1259/bjr.20170022

29 Lo PC, Dahlberg SE, Nishino M, Johnson BE, Sequist LV, Jackman DM, Jänne PA and Oxnard GR: Delay of treatment change after objective progression on first-line erlotinib in epidermal growth factor receptor-mutant lung cancer. Cancer 121(15): 2570-2577, 2015. PMID: 25876525. DOI: 10.1002/ cncr.29397

30 Gomez DR, Blumenschein GR Jr, Lee JJ, Hernandez M, Ye R, Camidge DR, Doebele RC, Skoulidis F, Gaspar LE, Gibbons DL, Karam JA, Kavanagh BD, Tang C, Komaki R, Louie AV, Palma DA, Tsao AS, Sepesi B, William WN, Zhang J, Shi Q, Wang XS, Swisher SG and Heymach JV: Local consolidative therapy versus maintenance therapy or observation for patients with oligo metastatic non-small cell lung cancer without progression after first-line systemic therapy: a multicentre, randomised, controlled, phase 2 study. Lancet Oncol 17: 16721782, 2016. PMID: 27789196. DOI: 10.1016/S14702045(16)30532-0 